\title{
A search for cyclical sources of $\gamma$-ray emission on the period range from days to years in the Fermi-LAT sky
}

\author{
D. A. Prokhorov ${ }^{1 \star}$ and A. Moraghan ${ }^{2}$ \\ 1 School of Physics, Wits University, Private Bag 3, WITS-2050, Johannesburg, South Africa \\ 2 Academia Sinica Institute of Astronomy and Astrophysics, P.O. Box 23-141, Taipei 106, Taiwan
}

Accepted ..... Received .....; in original form .....

\begin{abstract}
A systematic search for cyclical sources of $\gamma$-ray emission on the period range from days to years in the Fermi-LAT sky is performed. Looking for cyclical emission, the sky is binned into equal-area pixels and the generalised Lomb-Scargle periodogram is computed for each of these pixels. The search on the period range between 2.5 and 30 days in the Galactic plane confirms periodicities of three binaries, LSI $+61^{\circ} 303$, LS 5039, and 1FGL J1018.6-5856. The all-sky search on the period range between 30 days and 2.5 years confirms periodicities of three blazars, PG 1553+113, PKS 2155304, and BL Lacertae. Evidence for periodic behaviours of four blazars, 4C +01.28 , S5 0716+71, PKS 0805-07, and PKS 2052-47, are presented. Three of these blazars, $4 \mathrm{C}+01.28$, PKS 0805-07, and PKS 2052-47, are located at high redshifts. These three sources are potential candidates to binary systems of supermassive black holes provided that major galaxy mergers are more frequent and that galaxies are more gas-rich at high redshifts.
\end{abstract}

Key words: gamma-rays: general, radiation mechanisms: non-thermal

\section{INTRODUCTION}

Two classes of sources are known to be cyclical emitters in the $\mathrm{HE}(0.1-100 \mathrm{GeV}) \gamma$-ray regime on the period range from days to years. The first class consists of binaries showing orbitally modulated $\gamma$-ray emission, including LS 5039 (3.9 day period, Abdo et al. 2009b), 1FGL J1018.6-5856 (16.6 days, Ackermann et al. 2012b), LS I $+61^{\circ} 303$ (26.5 days, Abdo et al. 2009a) in the Milky Way, and CXOU J053600.0-673507 (10.3 days, Corbet et al. 2016) in the Large Magellanic Cloud. The second class consists of blazars, showing strong evidence for quasi-periodic modulations in $\gamma$ rays, including PG $1553+113$ (2.2 year period, Ackermann et al. 2015a), PKS 2155-304 (1.7 years, Sandrinelli et al. 2014; Zhang et al. 2017), and BL Lacertae (1.9 years, Sandrinelli et al. 2017). Modulated $\gamma$-ray signals from these sources were detected by Fermi-LAT. This pairconversion $\gamma$-ray space-based telescope (Atwood et al. 2009) normally operates in sky-survey mode where the whole sky is observed every $3 \mathrm{hr}$. It provides a regular and uniform view of $\gamma$-ray sources allowing us to monitor binaries and blazars on a daily basis. Multi-wavelength observations provide independent confirmations of periodic signals. Thus, the $\gamma$-ray binaries also emit signals with the same periods at other

\footnotetext{
* E-mail:phdmitry@gmail.com
}

frequencies (e.g., periodic $\mathrm{TeV}$ emission with a 3.9 day period from LS 5039 detected by H.E.S.S., see Aharonian et al. 2006), while the blazar, PG $1553+133$, emits an optical signal of period consistent with that detected in $\mathrm{HE} \gamma$ rays Ackermann et al. 2015a).

Hundreds of interacting binaries are X-ray emitters (e.g., Liu et al. 2006, 2007), but very few binaries produce detectable $\gamma$-ray emission. Detected $\gamma$-ray binaries are systems composed of a massive ( $>10$ solar masses) star and a compact object. The latter is either a neutron star or a black hole. The periods determined from $\mathrm{HE} \gamma$-ray data are compatible with binary periods obtained from radial velocity measurements (e.g. Aragona et al. 2009). Physical mechanisms responsible for periodic $\mathrm{HE} \gamma$-ray radiation likely invoke inverse Compton (IC) scattering from HE electrons or electron-positron pairs (for a review, see Dubus 2013). Target photons for IC processes are provided by the massive star and their radiation density varies along the eccentric orbit. HE electrons accelerated in the vicinity of the compact object up-scatter the stellar radiation. Since the angle at which an observer sees the star and $\mathrm{HE}$ electrons changes with the orbit, orbital modulation is expected.

$\gamma$-ray blazars are the most numerous type of $\gamma$-ray sources Acero et al. 2015, the Fermi-LAT 3FGL cata$\log$ includes $>1500$ blazars). However, most of the $\gamma$-ray blazars show erratic variability on a wide range of timescales 
(Abdollahi et al. 2016) without any evidence for periodicity. Blazars are active galactic nuclei (AGN) emitting a relativistic jet that is pointing very nearly along Earth's line of sight. At HE energies, blazar emission is often satisfactorily modelled as IC scattering of seed photons by HE electrons. Seed photons can be synchrotron photons produced in the jet itself (Maraschi et al. 1992; Bloom \& Marscher 1996) or photons from an external source, such as the accretion disk, broad-line region, or dusty infrared torus (Sikora et al. 1994). A temporal analysis plays an important role in studying the origin of $\gamma$-ray emission from blazars. In some cases, the separation of $\gamma$-ray events recorded during AGN flares from those during quiescent states can be used as a method of disentangling contributions from various emission components (e.g., Prokhorov \& Moraghan 2016). Various types of time variabilities in blazars are conceivable. Periodic $\gamma$-ray emission from blazars is expected if supermassive binary black holes are present at their centres (e.g., Rieger 2007). The geometrical origin of periodicity in $\gamma$-ray blazars, involving jet precession, intrinsic jet rotation, or helical structures in jets, is also possible (e.g., Rieger 2004; Mohan \& Mangalam 2015; Raiteri et al. 2015; Cavaliere et al. 2017; Sobacchi et al. 2017). Quasi-periodic fluctuations in the energy outflow efficiency in the magnetically arrested accretion regime (Tchekhovskov et al. 2011) suggest another potential mechanism for cyclic variability.

A supermassive black hole $(\mathrm{SMBH})$ is believed to reside at the centre of every large galaxy (e.g., Kormendy \& Richstone 1995). In hierarchical $\Lambda$ CDM cosmology, galaxies grow and evolve through mergers and accretion of smaller substructures (e.g., White \& Frenk 1991). If more than one of the merging galaxies contain an $\mathrm{SMBH}$, two or more SMBHs will be present in their resultant galaxy (e.g., Kulkarni \& Loeb 2012). Gravitationally bound binary SMBH systems are expected to be a product of mergers of galaxies and form when the separation between the SMBHs gradually shrinks (Begelman et al. 1980). A binary SMBH system can efficiently be formed in gas-dominated galaxies (e.g., Callegari et al. 2009). Since major mergers of galaxies are more frequent at high redshifts (Volonteri et al. 2009) when galaxies are also more gas-rich (Tacconi et al. 2010), the number of SMBH binaries increases rapidly with increasing redshift. SMBH binaries themselves are expected to be intrisically rare and a detection of quasi-periodic emission from a high-redshift blazar possibly modulated via the dynamics of gravitationally bound SMBHs can provide evidence for the existence of a population of sub-pc separation SMBH binaries (e.g., Bogdanović 2015). The previously claimed quasi-periodic $\gamma$-ray signals (that we also confirm) are from blazars, PG $1553+133(\mathrm{z}=0.360)$, PKS 2155-304 $(\mathrm{z}=0.116)$, and BL Lacertae $(\mathrm{z}=0.068)$, at low redshift 1 . In this paper, we pay our attention to cyclic $\gamma$-ray blazars during the early epoch of $0.7 \lesssim z \lesssim 2.0$.

The all-sky monitoring capability of Fermi-LAT is crucial to perform a search for new cyclical $\gamma$-ray sources. Using the Fermi-LAT data, we perform a systematic search for cyclical $\gamma$-ray binaries with periods between 2.5 days and 30 days in the Galactic plane, $-10^{\circ}<b<+10^{\circ}$, and a sys-

1 the redshifts of blazars are taken from the NASA/IPAC Extragalactic Database tematic all-sky search for cyclical $\gamma$-ray blazars with periods between 30 days and 2.5 years. To perform these investigations, the $\gamma$-ray sky is binned into equal-area pixels using the HEALPix package (Górski et al. 2005) and the generalised Lomb-Scargle (GLS) periodogram (Zechmeister \& Kürster 2009) is applied to establish periodic sources. The results of the search confirm the periodicities of $\gamma$-ray binaries LS 5039, 1FGL J1018.6-5856, and LS I +61 ${ }^{\circ} 303$, and $\gamma$-ray blazars PG 1553+113, PKS 2155-304, and BL Lacertae. In addition, new candidates in quasi-periodic $\gamma$-ray sources amongst blazars are found and include $4 \mathrm{C}+01.28$, PKS 0805-07, PKS 2052-47, and S5 0716+71. Three of these $\gamma$ ray sources, $4 \mathrm{C}+01.28$, PKS 0805-07, and PKS 2052-47, are located at high redshifts $(\mathrm{z}=0.890,1.837$, and 1.489 , respectively) and significantly more distant than PG $1553+113$, PKS 2155-304, and BL Lacertae. Therefore, the blazars, 4C +01.28, PKS 0805-07, and PKS 2052-47, are potential candidates to binary $\mathrm{SMBH}$ systems. Our systematic analysis is also a powerful tool to separate astrophysical periodic $\gamma$-ray signals from the known instrumental effects, in addition to effects caused by the Sun and the Moon moving through the sky, that all lead to an extrinsic $\gamma$-ray modulation.

\section{FERMI-LAT OBSERVATIONS AND TIME SERIES ANALYSIS}

The Fermi satellite was launched on 2008 June 11 into a nearly circular Earth orbit with an altitude of $565 \mathrm{~km}$ and an inclination of $25.6^{\circ}$. The orbital period is 96.5 minutes, and the orbit has a precession period of 53.4 days (so the RA and Dec of the orbit poles trace a 25.6 degree circle on the sky every 53.4 days). The Fermi satellite has two instruments onboard, the Large Area Telescope (LAT) pair-conversion detector and the Gamma Ray Burst Monitor (GBM). The Fermi-LAT instrument (Atwood et al. 2009) provides coverage over the energy range from $\sim 20 \mathrm{MeV}$ to several hundreds of $\mathrm{GeV}$. It has a large field of view ( $\approx 20 \%$ of the sky), and has been scanning the entire sky since it began routine science operation on 2008 August 4. It provides an angular resolution per single event of $5^{\circ}$ at $100 \mathrm{MeV}$, narrowing to $2^{\circ}$ at $300 \mathrm{GeV}$, and further narrowing to $0.15^{\circ}$ at $10 \mathrm{GeV}$ Atwood et al. 2013).

The Fermi-LAT Pass 8 data is downloaded from the Fermi Science Support Center 2 . The data acquired during each week of the Fermi's science mission are contained in weekly data files. Pass 8 is an event-level reconstruction analysis framework (Atwood et al. 2013) applied to the data taken by the Fermi-LAT, which results in an increase in $\gamma$-ray acceptance by $20-40 \%$ with respect to the previous Pass 7 reprocessed data release. For the data analysis, the Fermi Science Tools v10r0p5 packag $3^{3}$ and P8R2_SOURCE_V6 instrument response functions are used. Pass 8 SOURCE class photon data (evclass=128) spanning 7.8 years (MET 239557417 - 485913604) with energies between $300 \mathrm{MeV}$ and $500 \mathrm{GeV}$ are selected. The SOURCE event class is tuned to balance statistics with background flux

\footnotetext{
2 http://heasarc.gsfc.nasa.gov/FTP/fermi/data/lat/ weekly/photon/

3 https://fermi.gsfc.nasa.gov/ssc/data/analysis/ software/
} 
for long-duration point source analysis. By excluding events with reconstructed energies below $300 \mathrm{MeV}$, we tighten the point spread function (PSF). Contamination from the $\gamma$ ray-bright Earth's limb is avoided by removing all events with zenith angle $>90^{\circ}$. The recommended quality cuts (DATA QUAL==1 \&\& LAT CONFIG==1) are applied. The data is binned into time intervals of 12 hours and is binned using the HEALPix package into a map of resolution $N_{\text {side }}=32$ in Galactic coordinates with 'RING' pixel ordering. With these settings, the total number of pixels is equal to 12288 and the area of each pixel is $3.4 \mathrm{sq}$. deg. The resolution of the map is chosen according to the size of the Fermi-LAT PSF above $300 \mathrm{MeV}$. To compute the exposure for each pixel, the standard tools gtltcube and gtexpcube 2 are used. To correct the livetime for the zenith angle cut, the "zmax" option on the command line is used. For each pixel and each time interval, the number of photons are counted, the corresponding value of exposure is computed, and the integral $\gamma$-ray flux is calculated. Fermi-LAT light curves are created through aperture photometry to provide a model independent measure of the flux. The time bins used in this paper are sufficiently long (of 12 hours at least) and, therefore, barycenter corrections of the arrival times of the $\gamma$-ray photons are neglected. The search for cyclical $\gamma$-ray emission in binned equal-area portions of the sky has the advantage that it minimises the number of assumptions required for quasi-periodicity searches (e.g., it does not rely on both the distribution of $\gamma$-ray sources in the sky and the foreground diffuse model). However, the minimisation of the assumptions in turn leads to a decrease in the sensitivity of the method due to the possible leakage of $\gamma$ rays from a source to neighbouring pixels and due to the contamination of a $\gamma$-ray signal from the strongest $\gamma$-ray source in the pixel by other $\gamma$-ray sources belonging to the same pixel.

The Lomb-Scargle periodogram is a common statistical tool used in time series analysis to search for periodicities Lomb 1976; Scargle 1982). The problem in question is to establish the existence of a periodic signal, despite the presence of noise, where noise is random variations in the source flux. The method is equivalent to fitting sinusoidal waves, $y=\alpha \cos (\omega t)+\beta \cos (\omega t)$, in searches for periodic $\gamma$-ray signals. In this paper, the GLS periodogram (Zechmeister \& Kürster 2009) is used. Compared with the Lomb-Scargle periodogram, the GLS periodogram takes the measurement errors into account. It also introduces an offset, $c$, resulting in a generalisation of the periodogram to the equivalent of fitting a sinusoidal wave plus a constant, i.e., $y=\alpha \cos (\omega t)+\beta \cos (\omega t)+c$. In the context of Galactic $\gamma$-ray binaries, the diffuse Galactic $\gamma$-ray emission produced through pion decay Ackermann et al. 2012a) contributes to a constant term, whereas in the context of $\gamma$-ray blazars, it is the isotropic diffuse $\gamma$-ray background Ackermann et al. $2015 \mathrm{~b}$ ) that contributes to a constant term. The known $\gamma$-ray binaries have periods between 3.9 days and 26.5 days and the known cyclic $\gamma$-ray blazars have periods between 1.7 years and 2.2 years, and therefore enclose many 12 -hour time intervals. Time bins of a 12-hour and 1-day duration are used to search for $\gamma$-ray binaries in the Galactic plane, $-10^{\circ}<b<+10^{\circ}$. Longer time bins of a 3-day, 7-day, and 14-day duration are used for the all-sky search for cyclic $\gamma$-ray blazars. These time bins contain a whole number of 12-hour intervals. Typically, the average number of $\gamma$-ray events per time bin is $>10$. In this paper, the python module, astromi 4 (VanderPlas et al. 2012), is used for a time series analysis. The GLS periodograms are computed and the positions of peaks in the GLS periodograms are obtained using the tool, astroML.time_series.lomb_scargle. The chance probability of finding a peak in the GLS periodogram higher than the observed value is also estimated using this tool and is cross-checked using an alternative method, astroML.time_series.lomb_scargle_bootstrap. Both these estimates of the chance probability are computed under a condition of the exclusive presence of white (i.e., flat-spectrum) noise. $\gamma$-ray light curves of blazars are generally characterised by power-law noise with the power spectrum density (PSD) decreasing with frequency (e.g., Abdo et al. 2010; Nakagawa \& Mori 2013; Sobolewska et al. 2014). In this case, the chance probability is lower than that for the white noise distribution because of more power in longer time scales. Therefore, firstly we compute the chance probability under a condition of white noise for each pixel in the sky; secondly comparing the computed chance probability with the probability threshold we select pixels with possible $\gamma$-ray cyclic candidate sources; and thirdly if the candidate source is associated with a blazar, we calculate the power spectrum density of the source and perform simulations to check reliability of the source quasi-periodicity.

\section{RESULTS OF THE SEARCH FOR CYCLICAL $\gamma$-ray SOURCES}

In this Section, the results of the search for $\gamma$-ray sources in the Fermi-LAT sky are presented. To establish a periodicity of a $\gamma$-ray source, the following requirements must be satisfied. The highest observed peak in the GLS periodogram must have a very small probability to be found by chance under a condition of white noise. In other words, the chance probability of $<5.7 \times 10^{-7}$, corresponding to a $>5 \sigma$ confidence level, must be satisfied for detection of periodiclike behaviour. Furthermore, to ensure that the conclusion on a source periodicity does not depend on the bin width of the light curve, the periodicity detection found using a one time binning scheme must be confirmed using an alternative time binning scheme, and the periods found using these two different time binning schemes must be consistent. To be associated with a $\gamma$-ray source, a pixel showing a periodic-like behaviour must contain a source from the Fermi-LAT 4-year point source (3FGL) catalog. The associated $\gamma$-ray sources are additionally analysed by selecting $\gamma$ rays within a $1^{\circ}$ radius (and also within a more extended region of a $2^{\circ}$ radius) from the 3FGL source position and at energies above $300 \mathrm{MeV}$. The additional analyses by means of the GLS periodogram must also show the presence of a periodicity above a $5 \sigma$ level (under a condition of white noise) in order to establish a periodicity of the signal from the 3FGL source. Using 150000 bootstrap resamplings, the presence of a periodicity of the associated $\gamma$-ray source at $\mathrm{a}>4 \sigma$ confidence level is cross-checked. If the associated $\gamma$-ray source is a blazar, then we compute its PSD by 
means of the astroML.fourier.PSD_continuous tool, fit the computed PSD with a power-law function to calculate the power-law index, and use simulations to assess the chance probability to observe the corresponding peak in its GLS periodogram under a condition of power-law noise by means of the astroML.time_series.generate_power_law tool. Finally, a check if a periodic-like behavior of the source can be caused by the known instrumental effects or periodic $\gamma$-ray background is performed. The $\gamma$-ray sources satisfying all these requirements are shown in Table 1. The 1st column of Table 1 shows the pixel number according to the 'RING' pixel ordering, the 2 nd column shows the signal period in units of days, the 3rd and 4th columns show the name of the associated sources and its name in the 3FGL catalog. The 5 th column shows the source type. The 6 th shows the chance probability that the detected periodic signal belongs to the white (flat) noise. The 7th column shows the best-fit value of the PSD slope. The 8 th shows the chance probability that the detected periodic signal belongs to the noise with the given power-law PSD. The results of searches for $\gamma$ ray binaries and blazars are described in the next two paragraphs. A short discussion about the instrumental effects is given at the end.

The analysis performed on the period range between 2.5 and 30 days shows that the pixels with numbers 6000 and 6214 satisfy all the above requirements. These two pixels contain $\gamma$-ray sources, LSI $+61^{\circ} 303$ and LS 5039. The $\gamma$-ray flux above $300 \mathrm{MeV}$ (taken from the 3FGL catalog) from LSI $+61^{\circ} 303$ is fourteen times higher than that of the other 3FGL source in the former pixel, while the flux above 300 $\mathrm{MeV}$ from LS 5039 is eleven times higher than the sum of fluxes of the two other sources belonging to the latter pixel. Therefore, we identify LSI $+61^{\circ} 303$ and LS 5039 with $\gamma$-ray cyclic emission in these two pixels. The computed periods, 27.0 and 3.9 days, are consistent with those reported by Abdo et al. (2009a, b) . Only these two pixels in the Galactic plane show a periodic behaviour at a $>5 \sigma$ confidence level on this period range in two different time binning schemes (12-hour and 1-day time bins). The periodic behaviour of these two binaries was previously established in $\gamma$ rays during the 1st year of the Fermi-LAT mission. The pixel with number 6309 is the only other pixel in the Galactic plane showing a periodic behaviour at a $>4 \sigma$ confidence level in different time binning schemes. This pixel contains the known $\gamma$-ray binary, 1FGL J1018.6-5856. The highest peak for the pixel number 6309, corresponds to a period of 16.5 days which is consistent with that found for binary, 1FGL J1018.6-5856, by Ackermann et al. (2012b). The additional analysis of $\gamma$ rays within a $1^{\circ}$ radius from the position of the source 3FGL J1018.9-5856 contained in this pixel (and corresponding to 1FGL J1018.6-5856), at energies above 300 $\mathrm{MeV}$, results in a larger periodogram peak, shows that the chance probability that the detected periodic signal belongs to the noise decreases from $0.001 \%$ to $7.0 \times 10^{-7} \%$, and therefore shows a periodic behaviour at a $>5 \sigma$ confidence level. This binary is also included in Table1 The pixel containing this binary also contains six other 3FGL sources and the ratio of the flux above $300 \mathrm{MeV}$ from 3FGL J1018.9-5856 to the sum of the other sources' fluxes is about 0.8 . Therefore, centering of the aperture at the binary position increases the significance of a periodic behaviour. Similarly, the additional analysis of $\gamma$-ray emission from LSI $+61^{\circ} 303$ shows that the chance probability that the detected periodic signal belongs to the noise decreases from $4.0 \times 10^{-7} \%$ to $<1.0 \times 10^{-10} \%$ when the aperture with a $1 \mathrm{deg}$ radius centred on the source is used. The performed search confirms the previous results about periodicities of three $\gamma$-ray binaries belonging to the Galactic plane. No new candidates to periodic $\gamma$-ray sources with properties similar to these binaries is established in the Galactic plane.

The analysis performed on the period range between 30 days and 2.5 years shows that seven pixels with numbers 1269, 1867, 3187, 4753, 7265, 10173, and 10992 satisfy all the periodicity requirements. These seven pixels contain seven $\gamma$-ray blazars. For five of these pixels there is a oneto-one correspondence between pixels and 3FGL sources, while the remaining two pixels, 1269 and 4753, contain two 3FGL sources each. One of the 3FGL sources in each of these pixels is a strongly dominant contributor to the total flux from the pixel above $300 \mathrm{MeV}$ (with a fraction of $90 \%$ and $97 \%$, respectively). It allows us to avoid ambiguity in quasi-periodic source identification. Three of these blazars are PG 1553+113, BL Lacertae, and PKS 2155-304, whose $\gamma$-ray periodicities were previously reported in the literature. The computed periods (see Table 1) agree with those previously reported. These three blazars show the highest statistical chances to be periodic $\gamma$-ray sources. The four other blazars, 4C +01.28, S5 0716+71, PKS 0805-07, and PKS 2052-47, are new candidates to quasi-periodic $\gamma$-ray blazars. Three of the four candidates are located at high redshifts of $\mathrm{z}=0.89(4 \mathrm{C}+01.28), 1.84$ (PKS 0805-07), and 1.49 (PKS 2052-47), and are much more distant than the previously reported quasi-periodic $\gamma$-ray blazars. Taking that binary SMBH systems are expected to be more frequent at high redshifts into account, these distant blazars are potential candidates to binary systems of SMBHs. The fourth candidate is S5 $0716+71$ and is located at redshift of 0.31 . The LS periodogram analysis of the optical data for this blazar reveals two significant peaks at 359 and 63 days (Rani et al. 2013). The former peak is at the frequency close to the frequency of the peak in the GLS periodogram of the $\gamma$-ray data. But it is hard to provide strong, independent, supporting evidence in favour of a quasi-periodic behaviour at this frequency due to limited number of observed cycles in the optical data Rani et al. 2013). To illustrate possible quasi-periodic behaviours of the three new high-redshift candidates, the light curves are binned into time intervals of width one tenth of the corresponding source period. Figure 1 shows cyclical variations of the measured flux (i.e., counts/exposure) as a function of the phase for each of these $\gamma$-ray blazars. The average flux within each phase derived from the Fermi-LAT observations is overplotted as a dashed line. This figure also shows the GLS periodograms for these sources. Although, cyclic behaviors of these four candidates are less statistically significant than those of PG $1553+113$, BL Lacertae, and PKS 2155-304 (see Table 1), multi-wavelength analyses of these quasi-periodic source candidates are of interest to check if periodic-like signals are present at other frequencies. Taking the periods of the seven blazars showing the possible quasi-periodic behaviours into account, one can expect to observe their next several $\gamma$-ray emission cycles in the coming five years. The locations of these ten sources (three binaries + seven blazars), showing periodic-like $\gamma$-ray emission in the sky are visualised in Fig. 2 
Table 1. Cyclical sources of HE $\gamma$-ray emission. The values shown in bold type are obtained from a more sensitive analysis of the 3FGL source belonging to the pixel.

\begin{tabular}{|c|c|c|c|c|c|c|c|}
\hline $\begin{array}{c}\text { Pixel } \\
\text { number }\end{array}$ & $\begin{array}{c}\text { Period } \\
\text { (days) }\end{array}$ & Source name & 3FGL name & Type & $\begin{array}{c}\text { Probability } \\
\text { (white noise) }\end{array}$ & PSD slope & $\begin{array}{c}\text { Probability } \\
\text { (power-law noise) }\end{array}$ \\
\hline 1269 & 445 & 4C +01.28 & J0158.5+0133 & blazar & $3 \times 10^{-7} \%$ & 0.64 & $\mathbf{0 . 9 \%}$ \\
1867 & 798 & PG $1553+113$ & J1555.7+1113 & blazar & $<1 \times 10^{-10 \%}$ & 0.53 & $<0.1 \%$ \\
3187 & 346 & S5 0716+71 & J0721.9+7120 & blazar & $4 \times 10^{-10 \%}$ & 0.57 & $0.1 \%$ \\
4753 & 658 & PKS 0805-07 & J0808.2-0751 & blazar & $2 \times 10^{-7} \%$ & 0.77 & $6.7 \%$ \\
6000 & 27.0 & LSI +61 303 & J0240.5+6113 & binary & $<1 \times 10^{-10 \%}$ & - & - \\
6214 & 3.9 & LS 5039 & J1826.2-1450 & binary & $<1 \times 10^{-10 \%}$ & - & - \\
6309 & 16.5 & 1FGL J1018.6-5856 & J1018.9-5856 & binary & $7 \times 10^{-7 \%}$ & - & - \\
7265 & 698 & BL Lacertae & J2202.7+4217 & blazar & $1 \times 10^{-10 \%}$ & 0.74 & $0.9 \%$ \\
10173 & 637 & PKS 2052-47 & J2056.2-4714 & blazar & $3 \times 10^{-10 \%}$ & 0.69 & $\mathbf{0 . 4 \%}$ \\
10992 & 644 & PKS 2155-304 & J2158.8-3013 & blazar & $<1 \times 10^{-10} \%$ & 0.67 & $0.2 \%$ \\
\hline
\end{tabular}

To assess the chance probability to observe the corresponding peaks in the GLS periodograms of the seven blazars under a condition of power-law noise, we calculated the PSD for these sources for the Fermi-LAT weekly light curves. Using the optimize.leastsq tool from the SciPy library, we applied least-square fits to the PSD assuming a power-law PSD $\propto \nu^{-\alpha}$, where $\nu$ is the frequency. The best-fit values of the PSD slopes are shown in Table 1 For PKS 2155-304 and BL Lacertae, the best-fit PSD slope values of 0.67 and 0.74 , respectively, are compatible with $0.58 \pm 0.33$ and $0.41 \pm 0.47$, reported by Nakagawa \& Mori (2013), and with $0.64_{-0.50}^{+0.79}$ and $0.93_{-0.14}^{+0.18}$, reported by Sobolewska et al. (2014). We use the astroML.time_series.generate_power_law tool based on the algorithm from Timmer \& Koenig 1995) to simulate power-law noise light curves with the given PSD slope. For each of the simulated light curves, we compute the GLS periodogram. We calculate the chance probabilities to observe the peaks in the GLS periodograms of the seven blazars by comparing their power peak values with those obtained in these simulations. The calculated chance probabilities that the detected periodic signal belongs to the power-law noise are shown in Table 1. This analysis shows that the power peaks in the periodograms of PG 1553+133, PKS 2155-304, and BL Lacertae have $<1 \%$ chance to be caused by a statistical fluctuation. For the three high-redshift candidates to quasi-periodic $\gamma$-ray sources, the power peaks of $4 \mathrm{C}+01.28$ and PKS 2052-47 have $2.7 \%$ and $1.0 \%$ chance to be caused by a statistical fluctuation, while the chance of the power peak of PKS 0805-07 is 6.7\%. Though the chance of the power peak of PKS $0805-07$ is above $5 \%$, this $\gamma$-ray blazar is the most distance source (at $\mathrm{z}=1.837$ ) amongst the four new candidates. The accumulation of more $\gamma$-ray data is needed to re-access its quasi-periodicity. We checked and found that the results obtained from equal-area portions binned with HEALPix and containing these sources, and from circular regions of $1^{\circ}$ (or $2^{\circ}$ ) radius around these sources are consistent.

To reduce uncertainty caused by both the diffuse background and background due to the presence of $\gamma$-ray sources projected near the new candidates to cyclic $\gamma$-ray blazars, we performed a likelihood analysis of these four blazars using the standard Fermi Science Tools package. We selected events from $300 \mathrm{MeV}$ to $500 \mathrm{GeV}$ in a circular region of interest of $20^{\circ}$ radius centred on the position of each of the blazars. The data was binned in thirty equal logarithmically spaced energy intervals. To model the Galactic and isotropic background diffuse emission, we used the tem- plates, gl1_iem_v06 and iso_P8R2_SOURCE_V6_v06. The other cuts applied to the Fermi-LAT data are identical to those used in Sect. 2. We included $\gamma$-ray sources from the 3FGL catalog to model the data within the regions of interest. A binned maximum likelihood model fit method was applied to each weekly time bin to derive the light curves of the integrated flux $(\mathrm{E}>300 \mathrm{MeV})$. We computed the GLS periodograms of these sources and found that their power peaks are at 442 days for $4 \mathrm{C}+01.28$, at 340 days for $\mathrm{S} 50716+71$, at 676 days for PKS 0805-07, and at 642 days for PKS 205247. These positions of the GLS power peaks are compatible with those obtained above (see Table 1). Three of these four power peaks are significant at $>5 \sigma$ confidence level under a condition of white noise, while the power peak of PKS 0805-07 is significant at $4.7 \sigma$ (corresponding to the chance probability of $3 \times 10^{-4} \%$ ) under this condition. We also calculated the PSD slopes for these sources and found that the PSD slope values are compatible with those are shown in Table 1 Finally, we computed the chance probability to observe the power peaks in the GLS periodograms under a condition of power-law noise and found that $4 \mathrm{C}+01.28$ has $0.9 \%$ chance of being a statistical fluctuation and PKS 2052-47 has $0.4 \%$ chance of being a statistical fluctuation. This strengthens the results obtained from the analysis of equal-area portions binned with HEALPix.

Other pixels showing periodic behaviours which are not attributed to a $\gamma$-ray source will be discussed. Firstly, the pixel number 10889 satisfies most of the requirements, but does not contain a source from the 3FGL catalog. The results show that the 656-day periodicity of the $\gamma$-ray signal from pixel 10889 is due to its neighbouring pixel, 10992, which contains the strong $\gamma$-ray source, PKS 2155-304, whose period is about 644 days. Secondly, the pixels with numbers 5381, 5509, 5510, 5637, 5638, 5765, 5892, 5893, 6021, 6302, $6429,6430,6557,6658,6684$, and 6786 , show periodic behaviours (with a period of about 53 days) caused by the modulation of the $\gamma$-ray signals at the precession period of the orbit of the Fermi spacecraft. Thirdly, the pixels with numbers 5509, 5636, 5766, 6083, 6173, 6430, and 6686, show periodic behaviours (with a period of about 91 days) caused by the modulation at a period of $1 / 4$ of a year. This modulation is expected for aperture photometry for positions in the sky close to the positions of bright sources. It results from the 4-fold symmetry of the Fermi-LAT PSF and a systematic change in spacecraft orientation during the course of a 

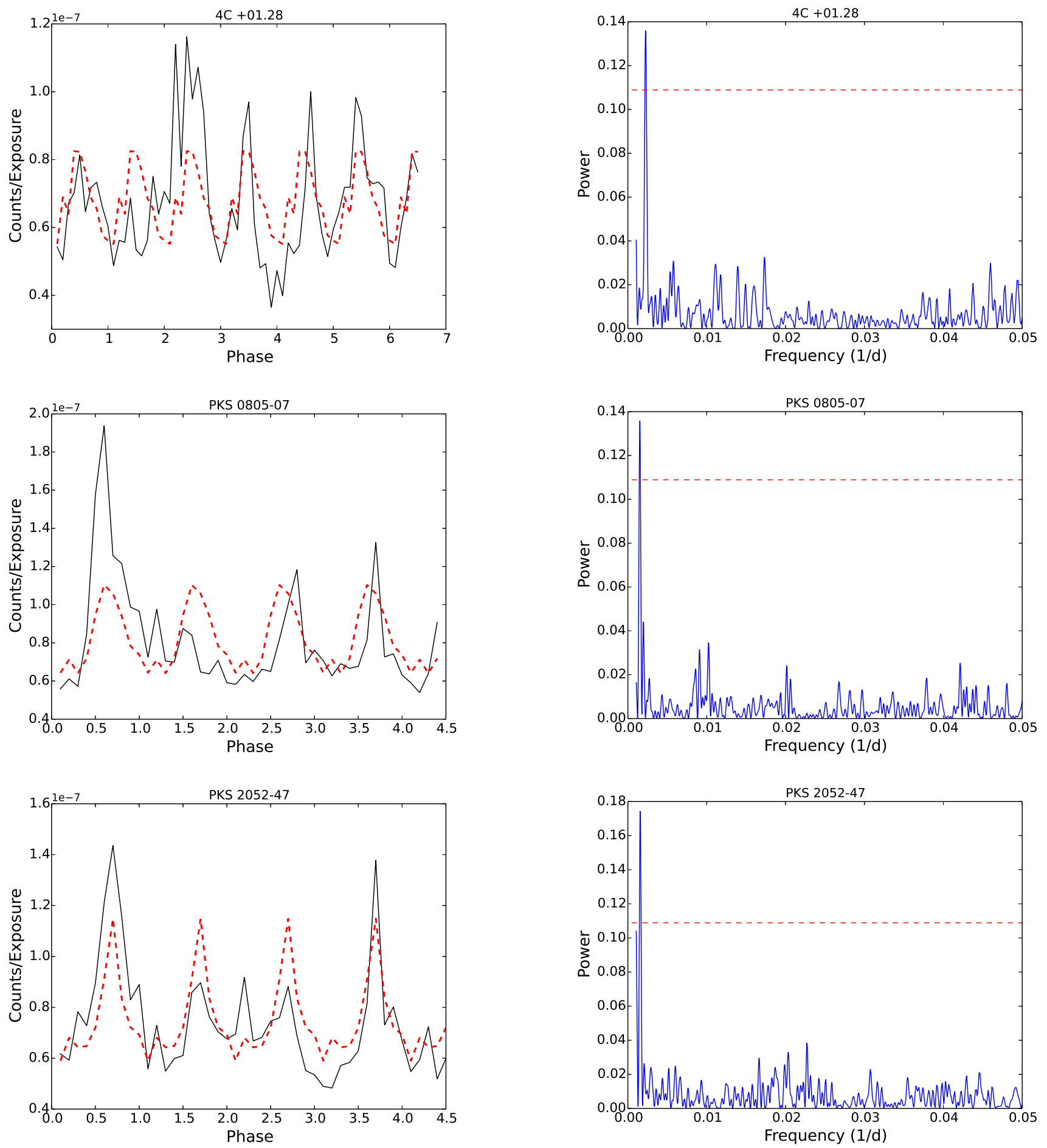

Figure 1. The left panel shows the periodic-like variations of the measured flux of the three high-redshift candidates to quasi-periodic $\gamma$-ray sources as a function of the phase (solid line) and the average flux within each phase (dashed line). The right panel shows the GLS periodogram for these sources, where the dashed line shows the level corresponding to the chance probability of $<5.7 \times 10^{-7}$ under a condition of white noise.

year 5 . The pixels showing modulated $\gamma$-ray signals due to these two instrumental effects are shown in Fig. 2 and are

5 https://fermi.gsfc.nasa.gov/ssc/data/analysis/LAT_ caveats_temporal.html concentrated near the positions of the Vela and Geminga pulsars. Lastly, no effects caused by the Moon (27.3-day period) and Sun (1-year period) moving through the sky are detected in this analysis. This is mostly owing to the choice of long time bins and small spatial bins used in this analy- 


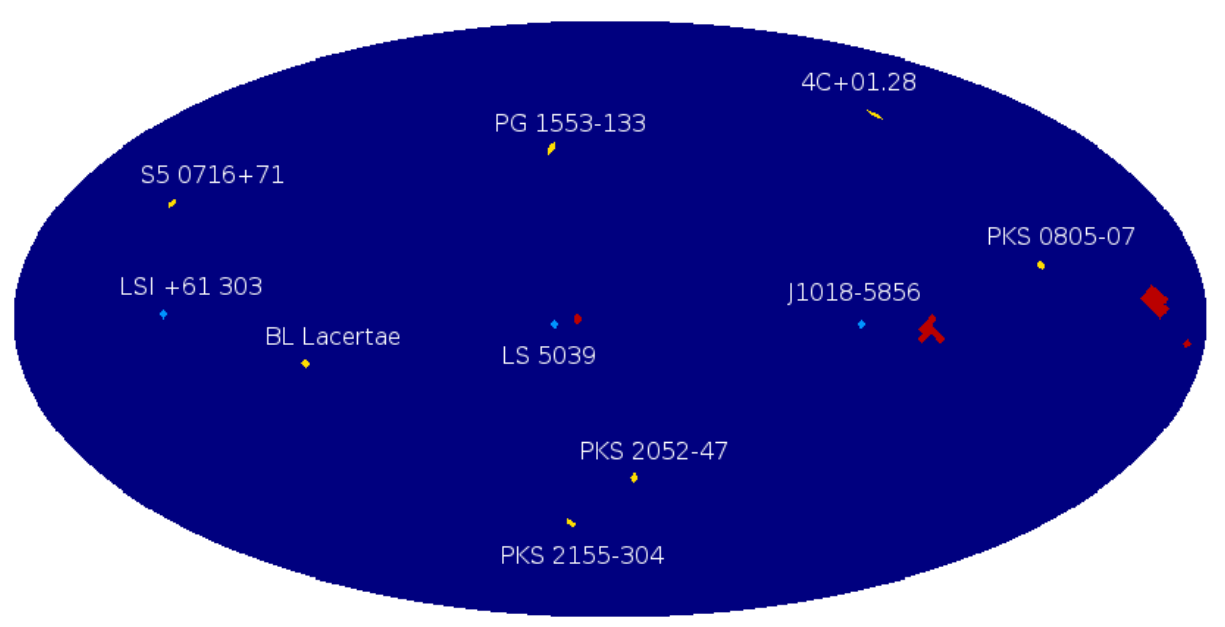

Figure 2. Figure shows periodic-like $\gamma$-ray sources in the Fermi-LAT sky. These include three binaries (the corresponding pixels are shown in blue) and seven blazars (shown in yellow). Modulated $\gamma$-ray signals from the pixels shown in red are due to instrumental effects, see the text for details. This figure can be seen in colour in the online version.

sis. To find more about the effect due to a $\gamma$-ray foreground modulation by the Moon, see Corbet et al. (2013). To check if the periodic $\gamma$-ray foreground due to the solar $\gamma$-ray emission (e.g., for a blazar of 3C 279, see Barbiellini et al. 2014) can affect the conclusion on periodicities of the seven blazars from Table 1 an additional check is performed. The closest projected distance from the Sun amongst these seven sources is $4.6^{\circ}$ for $4 \mathrm{C}+01.28$ (the 2 nd closest is $17^{\circ}$ for PKS 2155304). To check the possible influence of the Sun on $\gamma$-ray emission from the pixel containing $4 \mathrm{C}+01.28$, the time intervals when the solar centre is at a projected distance closer than 10 degree from this blazar are removed. The subtracted time intervals include about $5 \%$ of the entire data set. The result of an analysis of the data set after subtraction is compatible with the result reported in this paper.

\section{CONCLUSIONS}

Fermi-LAT capability to observe sources over the entire sky on a daily basis led to the discovery of orbitally modulated $\gamma$-ray emission with periods between 2.5 days and 30 days from several Galactic binaries. Moreover, continuous, ongoing Fermi-LAT all-sky survey started in the summer of 2008 plays a crucial role in studying long-term quasi-periodic variability of blazars. The discovered quasi-periodic $\gamma$-ray emission from the blazar, PG $1553+113$, with a 2.2 year period (Ackermann et al. 2015a) demonstrated the necessity of multi-year $\gamma$-ray monitoring campaigns of blazars.

In this paper, we performed a systematic search for cyclical sources of $\gamma$-ray emission. We binned the sky into equal-area pixels using the HEALPix package and computed the GLS periodograms in searching for cyclic $\gamma$-ray signals from the sources belonging to these pixels. Our analysis confirmed the modulated $\gamma$-ray signals from the three Galactic $\gamma$-ray binaries and also confirmed the previously claimed quasi-periodicity of $\gamma$-ray blazars, including PG $1553+113$, PKS 2155-304, and BL Lacertae. The quasi-periodicities of the last two blazars were claimed by Zhang et al. (2017); Sandrinelli et al. (2017) when our manuscript was in preparation. The advantage of the systematic search for cyclic sources is that this analysis does not require any preselection of sources. In addition to the previously claimed quasi-periodic $\gamma$-ray blazars, we found evidence for periodic behaviours of four other blazars, $4 \mathrm{C}+01.28$, S5 $0716+71$, PKS 0805-07, and PKS 2052-47. Three of these sources are located at high redshifts and are potential candidates to binary systems of supermassive black holes given that the number of such rare systems increases rapidly with redshift. We conclude that the coming five years of $\gamma$-ray observations will be decisive in studying the quasi-periodic variability of these blazars owing to the need for observations to capture their next several emission cycles.

\section{ACKNOWLEDGEMENTS}

We are grateful to the referee for the constructive suggestions that helped us to improve the manuscript. DAP acknowledges support from the DST/NRF SKA post-graduate bursary initiative. Computations were performed on the computational facilities belonging to the ALMA Regional Center Taiwan, Academia Sinica, Taiwan.

\section{REFERENCES}

Abdo A. A. et al., 2010, ApJ, 722, 520

Abdo A. A. et al., 2009a, ApJ, 701, L123

Abdo A. A. et al., 2009b, ApJ, 706, L56

Abdollahi S. et al., 2016, ArXiv e-prints

Acero F. et al., 2015, ApJS, 218, 23

Ackermann M. et al., 2015a, ApJ, 813, L41

Ackermann M. et al., 2015b, ApJ, 799, 86

Ackermann M. et al., 2012a, ApJ, 750, 3

Ackermann M. et al., 2012b, Science, 335, 189

Aharonian F. et al., 2006, A\&A, 460, 743

Aragona C., McSwain M. V., Grundstrom E. D., Marsh A. N., Roettenbacher R. M., Hessler K. M., Boyajian T. S., Ray P. S., 2009, ApJ, 698, 514

Atwood W. et al., 2013, ArXiv e-prints

Atwood W. B. et al., 2009, ApJ, 697, 1071 
Barbiellini G. et al., 2014, ApJ, 784, 118

Begelman M. C., Blandford R. D., Rees M. J., 1980, Nature, 287, 307

Bloom S. D., Marscher A. P., 1996, ApJ, 461, 657

Bogdanović T., 2015, in Astrophysics and Space Science Proceedings, Vol. 40, Gravitational Wave Astrophysics, Sopuerta C. F., ed., p. 103

Callegari S., Mayer L., Kazantzidis S., Colpi M., Governato F., Quinn T., Wadsley J., 2009, ApJ, 696, L89

Cavaliere A., Tavani M., Vittorini V., 2017, ApJ, 836, 220

Corbet R., Cheung C. C., Kerr M., Ray P. S., 2013, ArXiv e-prints

Corbet R. H. D. et al., 2016, ApJ, 829, 105

Dubus G., 2013, A\&A Rev., 21, 64

Górski K. M., Hivon E., Banday A. J., Wandelt B. D., Hansen F. K., Reinecke M., Bartelmann M., 2005, ApJ, 622,759

Kormendy J., Richstone D., 1995, ARA\&A, 33, 581

Kulkarni G., Loeb A., 2012, MNRAS, 422, 1306

Liu Q. Z., van Paradijs J., van den Heuvel E. P. J., 2006, A\&A, 455, 1165

Liu Q. Z., van Paradijs J., van den Heuvel E. P. J., 2007, A\&A, 469, 807

Lomb N. R., 1976, Ap\&SS, 39, 447

Maraschi L., Ghisellini G., Celotti A., 1992, ApJ, 397, L5

Mohan P., Mangalam A., 2015, ApJ, 805, 91

Nakagawa K., Mori M., 2013, ApJ, 773, 177

Prokhorov D. A., Moraghan A., 2016, MNRAS, 457, 2433

Raiteri C. M. et al., 2015, MNRAS, 454, 353

Rani B. et al., 2013, A\&A, 552, A11

Rieger F. M., 2004, ApJ, 615, L5

Rieger F. M., 2007, Ap\&SS, 309, 271

Sandrinelli A., Covino S., Treves A., 2014, ApJ, 793, L1

Sandrinelli A. et al., 2017, ArXiv e-prints

Scargle J. D., 1982, ApJ, 263, 835

Sikora M., Begelman M. C., Rees M. J., 1994, ApJ, 421, 153

Sobacchi E., Sormani M. C., Stamerra A., 2017, MNRAS, 465, 161

Sobolewska M. A., Siemiginowska A., Kelly B. C., Nalewajko K., 2014, ApJ, 786, 143

Tacconi L. J. et al., 2010, Nature, 463, 781

Tchekhovskoy A., Narayan R., McKinney J. C., 2011, MNRAS, 418, L79

Timmer J., Koenig M., 1995, A\&A, 300, 707

VanderPlas J. T., Connolly A. J., Ivezić Ž., Gray A., 2012, in Conference on Intelligent Data Understanding (CIDU), pp. $47-54$

Volonteri M., Miller J. M., Dotti M., 2009, ApJ, 703, L86

White S. D. M., Frenk C. S., 1991, ApJ, 379, 52

Zechmeister M., Kürster M., 2009, A\&A, 496, 577

Zhang P.-f., Yan D.-h., Liao N.-h., Wang J.-c., 2017, ApJ, 835,260 